Autor / Author

\section{ALFÉREZ SÁNCHEZ, Maria}

Universidad Francisco de Vitoria maria.alferez @ufv.es

DOI: https://doi.org/10.32466/eufv-rel.2020.7.609.113-120
RECiBido / RECEIVED

Aceptado / Acepted

13 de marzo de 2020

10 de junio de 2020

PÁginas / Pages

De la 113 a la 120

ISSN / ISSN

$2386-2912$

\title{
Memoria de la edición bilingüe y estudio introductorio de los Comentarios a la Secunda Secundae (t. III/ q, 66) de Fr. Francisco de Vitoria
}

Memory of the bilingual edition and introductory study of Comentarios a la Secunda Secundae (t. III/ q. 66) by Fr. Francisco de Vitoria

La labor docente que Francisco de Vitoria desarrollada a mediados del siglo xvı en la Universidad de Salamanca contribuye de manera significativa a la renovación teológica de su tiempo. A través de sus Relecciones y Lecturas, propone una nueva orientación que ahonda de lleno en los problemas de su tiempo. Sin embargo, aunque se han sucedido varias y cuidadas ediciones sobre las Relecciones, aún carecemos de una edición crítica de las Lecturas que considere todos los manuscritos disponibles. Por ello, proponemos un proyecto de edición sobre la cuestión 66, «De furto et rapina», que considere todos los manuscritos disponibles.

\section{\#Escuela de Salamanca-escolástica-Francisco de Vitoria-justicia-hurto}

The teaching labour that Francisco de Vitoria develops in the mid-16th century at the University of Salamanca contributes significantly to the theological renewal of his time. Through Relecciones and Lecturas, he poses a new angle which deeply looks into the 
problems of his time. Nevertheless, although there have been several and thorough editions of Relecciones, we still lack a critical edition of Lecturas that encompasses all manuscripts which may be available. Therefore, we propose an edition project on the 66th question «De furto et rapina» which takes account of all available manuscripts.

\#School of Salamanca-Scholastic-Francisco de Vitoria-Justicie-Robery

\section{Hipótesis}

Desde la segunda mitad del siglo xv y durante los dos siglos siguientes, de manera especial en el xVI, se produce en España una profunda renovación doctrinal en todas las ciencias humanísticas, particularmente en las teológicas y jurídicas, que tuvo sus focos principales en la antigua Universidad de Salamanca, en la más reciente de Alcalá de Henares y en las portuguesas de Évora y Coímbra.

En la Facultad de Teología de la Universidad de Salamanca, desarrolló Francisco de Vitoria su magisterio desde 1526 hasta 1546. Desde su cátedra, contribuyó de manera significativa a la renovación teológica mediante el restablecimiento del tomismo centrado los contenidos de la Summa Theologiae, en detrimento de las Sentencias ${ }^{1}$ de Pedro Lombardo. Esta nueva orientación llevaba consigo un enfoque eminentemente práctico que ahondaba de lleno en los problemas de su tiempo. El dominico tuvo que enfrentarse a la crisis provocada por el descubrimiento de América, a la desintegración de la Europa cristiana y al desequilibrio social de España. Su magisterio en la cátedra prima de Teología de la Universidad de Salamanca rompe con la tradición medieval. Como prueba, su tesis de la Communitas Orbis se adelantó a su tiempo hasta convertirse en utopía. De ahí deriva precisamente su mayor signo de actualidad.

La relevancia del tomo III de De justitia de los Comentarios a la Secunda Secundae está explicitada en la edición de Beltrán de Heredia (1934) sobre dicho tomo, al que dedica una introducción especial. La justificación de esta introducción viene dada por la preferencia que tanto escotistas, nominalistas y humanistas daban a los estudios éticos y jurídicos, como puede observarse en las diversas ediciones y comentarios de la Ética de Aristóteles. Se pone de manifiesto el hecho de que Vitoria contaba con alumnos de otras facultades: canonistas, juristas, médicos, etc., por lo que el estudio de su doctrina alcanza actualmente una mayor trascendencia. De hecho, como señala Carabias Torres (2015), una buena parte de los temas que abordó la Escuela de Salamanca se tratan a raíz de solicitudes previas de informes reales.

\footnotetext{
1 A pesar de esto, Francisco de Vitoria continúo comentando las distinctiones de las Sentencias de Pedro Lombardo como complemento a la III Pars de la Summa (Langella, 2015, p.19).
} 
La clave del pensamiento de las lecciones contenidas en De justitia es la visión clara del alcance intensivo y extensivo del derecho natural, en contraposición con el derecho positivo. Es decir, su valor consiste en el establecimiento de un derecho universal uno y único para todos. Con todo, en el marco de la filosofía moral, se propone un estudio de los Comentarios a la Secunda-Secundae de Francisco de Vitoria, a través de los manuscritos de sus alumnos. En concreto, se abordará el estudio y la edición de la cuestión 66, «De furto et rapina», en aras de completar el alcance del pensamiento hispánico del siglo xvı. Su elección viene condicionada por la rabiosa actualidad y el interés social que un estudio sobre estas cuestiones puede suponer en el tratamiento de los problemas morales de nuestro tiempo.

\section{Antecedentes y estado de la cuestión}

Como hito en la labor editorial sobre la Escuela de Salamanca, es preciso destacar la labor desempeñada por el dominico Vicente Beltrán de Heredia, a quien se debe el haber puesto de manifiesto la importancia de este pensador. Tras un proceso de investigación en los archivos europeos de sus textos, realizó la edición de sus Comentarios a la Secunda Secundae de la Summa Theologiae. Además, posteriormente publicó gran cantidad de ensayos y monografías que versan sobre este asunto. Por ejemplo, su obra Los manuscritos del Maestro fr. Francisco de Vitoria (1928) es punto de referencia obligado para los estudios del pensamiento clásico español. Asimismo, habría que citar la actividad que desarrolló Luis Alonso Getino, autor de una cuidada edición de las Relecciones (1933-1935). También, hay que destacar la edición de las Relectiones (1960) de Teófilo Urdanoz, que llevo a cabo un óptimo trabajo de tipo ecdótico y crítico. A finales de los años setenta, tuvo lugar un gran enriquecimiento del patrimonio textual de Francisco de Vitoria, gracias a la labor de Luciano Pereña $(1967 ; 1981)$ y el Corpus Hispanorum de Pace.

Ahora bien, a pesar de que las ediciones de las Relectiones son numerosas y cuentan con gran valor crítico, la edición de las lecturas es todavía hoy un trabajo pendiente para los estudiosos. En los últimos años, es digna de encomio la labor que ha llevado a cabo Simona Langella (2015) mediante la consulta del material inédito conservado en las bibliotecas de Madrid (Nacional, Real, El Escorial), Salamanca (Pontificia, General, San Esteban), Santander (Menéndez y Pelayo), Archivo de Indias (Sevilla) y la Biblioteca Apostólica Vaticana. La estudiosa realiza una edición crítica sobre el Comentario a la Prima Secundae de la Summa Theologiae, cc. 90-103. También es recalcable su labor de estudio bibliográfico sobre las fuentes primarias y la literatura científica más actual sobre la Escuela de Salamanca, la cual nos presenta un amplio espectro sobre el que fundamentar futuros estudios.

Además, contamos con trabajos comparativos entre algunas cuestiones de los Comentarios a la Secunda Secundae y las Relectiones correspondientes a los mismos. Este es el caso de Felipe Castañeda (2010). El equipo de la Universidad de los Andes, del que es coordinador, se ocupado de la traducción y el tratamiento crítico de la Relección sobre el homicidio y el comen- 
tario a la cuestión 64, «Sobre el homicidio». La obra se establece como base para el estudio del pensamiento filosófico-moral relacionado con las cuestiones de homicidio y suicidio.

\section{Nuestra edición}

Durante el magisterio de Vitoria en Salamanca, había dos tipos de producciones académicas: las Lecturas $^{2}$ y las Relecciones ${ }^{3}$. Como se ha enunciado anteriormente, mientras que contamos con una ingente literatura científica referida a las Relecciones, con ediciones críticas abundantes y de calidad, la edición de las Lecturas es todavía hoy un tema pendiente que solo se ha presentado de manera fragmentaria. El número de manuscritos inéditos relacionados con las lecturas escolásticas de los que disponemos es amplísimo. Como obra de referencia, contamos con la edición de Beltrán de Heredia (1939) a los Comentarios de la II-II, apoyada en la edición de un solo manuscrito, perteneciente a Francisco Trigo. Esta es, por tanto, la razón de esta edición que se plantea la publicación de una parte de las lecturas inéditas, contenidas en los manuscritos de los alumnos de Francisco de Vitoria, con el fin de esclarecer su influencia en la historia del renacimiento teológico español del siglo xvı. Abarcaremos una pequeña parte de este corpus, a partir de la edición crítica de la quaestio 66, «De furto et rapina», del tomo III de sus Comentarios a la Secunda Secundae.

La edición de estas lecturas ha exigido un análisis de clasificación cronológica y de autenticidad. Beltrán de Heredia (1939) expone una cronología de las Lecturas, de la que se extrae que, en lo que interesa a nuestro estudio, la quaestio 66 de la Secunda Secundae fue explicada durante los años 1526-1529/1534-1537. Nuestro trabajo únicamente recogerá los manuscritos académicos, es decir, aquellos que fueron confeccionados por quienes asistieron a las lecciones, y no los extraacadémicos, realizados con posterioridad a través de la colección y copia de escrituras teológicas en cartapacios. Nos decantamos, pues, por una lectura primitiva y no por la explicación procedente de una fuente derivada.

Del manuscrito 43 de la Universidad de Salamanca que recoge los apuntes del bachiller Francisco Trigo, afirma Beltrán de Heredia (1939) que «El texto del presente manuscrito está redactado con esmero y parece reproducir bastante fielmente las mismas palabras del maestro. Es claro, ordenado, extenso y tiene otras cualidades que le hacen ser el mejor que de las lecciones de Vitoria se han conservado». Según Beltrán de Heredia el manuscrito Ottoboniano latino 1015 recogería la docencia impartida en los cursos 1526-29 aunque podría haber sido completado con lecciones posteriores debido a la rúbrica que se encuentra en el folio 226 ,

\footnotetext{
${ }^{2}$ La palabra «lectura» se aplicaba fundamentalmente a la materia señalada para cada curso, y por extensión al comentario sobre la misma, redactado por el profesor para sus lecciones, a la exposición oral de ese comentario en el aula y también a las anotaciones tomadas por los estudiantes.

${ }^{3}$ Lecciones extraordinarias - como conferencias - que los profesores debían pronunciar en ciertas fechas del año lectivo, generalmente en días festivos, para favorecer la asistencia de alumnos y profesores.
} 
q57: «Incipit materia de justitia secundum magistrum fratrem Franciscum a Victoria, quam incepit 19 octobris de 1535 annis». De este manuscrito afirma el mismo estudioso: «Roma. Biblioteca Vaticana. Cod. Ottob. 1015 a y b; dos vols.; en 4º 455 folios. Proceden de la biblioteca del duque de Altemps, según se indica en la primera guarda. El tomo primero contiene la exposición de la secunda secundae hasta la q. 89 inclusive y el segundo que comienza en el folio 249 lo restante». Por su parte el códice 64 del Monasterio de Montserrat recoge las lecciones relativas a las cuestiones 57 (a. 2) a la 153 impartidas en los cursos 1534-15374.

Así pues, partiendo del texto base que utiliza Beltrán de Heredia (Cód. 43/SUV2a/ Biblioteca Universitaria de Salamanca), se ha realizado un trabajo ecdótico con las variantes y añadidos que se encuentren en los demás manuscritos disponibles. A saber:

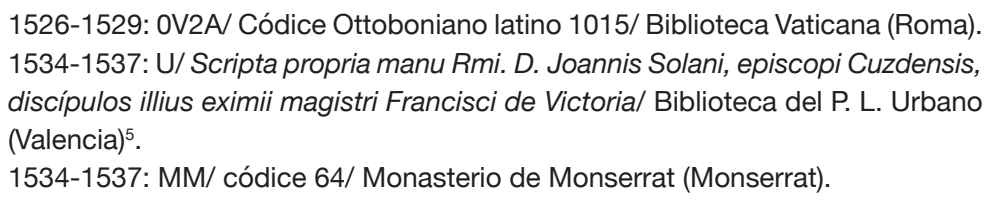

Con la intención de hacer el texto asequible a los investigadores y lectores interesados en el pensamiento hispánico, se presentará junto a la edición crítica del texto latino una traducción castellana lo más cuidada posible.

Por último, describimos someramente las características lingüísticas de los textos trabajados.

Los manuscritos de Monserrat y el Ottoboniano latino resultan hartamente complejos. Son varias sus peculiaridades. La primera de ellas es evidente: al no tratarse de documentos oficiales, no se ciñen a ninguna tipología de letra determinada, por lo que resulta complicado descifrar la letra del alumno en cuestión y las diversas abreviaturas o giros propios que se introducen. Por otro lado, a menudo se insertan dentro del texto latino expresiones coloquiales en español, las cuales intuimos que pueden ser del mismo Vitoria, dada su repetición en varios manuscritos ${ }^{6}$.

\section{Objetivos de esta edición}

1. Elaborar una edición crítica de la quaestio 66, perteneciente al t. III de los Comentarios a la Secunda Secundae de Francisco de Vitoria, integrando, sobre el texto base de Beltrán de Heredia, las variantes de los manuscritos aún inéditos.

\footnotetext{
${ }^{4}$ Este apartado aparecerá en el volumen Francisco de Vitoria sobre justicia, dominio y economía. Edición y contexto doctrinal de la cuestión «Sobre el hurto y la rapiña», que se publicará en 2020.

${ }^{5}$ Nos ha resultado imposible localizar este manuscrito, a pesar de haber realizado un pormenorizado trabajo de búsqueda bibliográfica.

${ }^{6}$ Por ejemplo, la expresión «es afrenta quitalle la capa», que destaca el carácter injurioso de la rapiña.
} 
2. Realizar un estudio introductorio sobre las cuestiones relativas al hurto encuadrándolas en el pensamiento clásico español del siglo xvı y conectándolas con las disputas filosófico-morales actuales ${ }^{7}$.

3. Acompañar el texto latino con una traducción al español, con el fin de favorecer su divulgación.

4. Iluminar el contenido de las Relecciones a partir del estudio de las Lecturas, para obtener una visión de conjunto a través de la cual sea posible calcular la influencia de la escolástica española.

5. La publicación: Francisco de Vitoria sobre justicia, dominio y economía. Edición y contexto doctrinal de la cuestión «Sobre el hurto y la rapiña».

La publicación Francisco de Vitoria sobre justicia, dominio y economía. Edición y contexto doctrinal de la cuestión "Sobre el hurto y la rapiña», a cargo de los doctores José Luis Cendejas Bueno y María Alférez Sánchez, pretende ofrecer a la comunidad científica los comentarios inéditos de Francisco de Vitoria a la cuestión 66 de la Secunda Secundae, que sirve para completar lo dicho sobre el dominio en la cc. 62.

El hurto y la rapiña se consideran pecados que atentan contra la justicia, es esta la perspectiva adoptada desde un punto de vista teológico. Vitoria no se limita a la mera repetición o resumen de los artículos de la Suma, sino que añade multitud de consideraciones del momento, probablemente conocidas por los estudiantes que asistían a sus lecciones.

Por nuestra parte, aunque sinteticemos aquí los artículos correspondientes de la Suma, recomendamos su lectura para apreciar el alcance del comentario vitoriano. De los tres manuscritos cuya traducción presentamos en este trabajo, en esta síntesis seguimos principalmente el de la Universidad de Salamanca del bachiller Francisco Trigo que resulta sin duda el más completo y detallado.

Adjuntamos a continuación el esquema que seguiremos en la citada publicación:

1. Francisco de Vitoria y su legado

2. Dos conceptos de derecho en Francisco de Vitoria

3. La justicia en los intercambios (I): la Escuela de Salamanca en el pensamiento económico

4. La justicia en los intercambios (II): el precio justo en Francisco de Vitoria

4.1. De la justicia a la teoría del precio justo

4.2. Teoría del precio justo

\footnotetext{
7 De estas cuestiones se ha encargado el profesor Dr. José Luis Cendejas Bueno, que junto con la autora del presente artículo, publicarán el volumen Francisco de Vitoria sobre justicia, dominio y economía. Edición y contexto doctrinal de la cuestión «Sobre el hurto y la rapiña» en el año 2020.
} 
5. La justicia en los intercambios (III): la usura según Francisco de Vitoria

5.1. La naturaleza del dinero como presupuesto del análisis sobre la usura

5.2. Si se peca al recibir interés por un préstamo en dinero

5.3. Si es lícito exigir otro beneficio por el dinero prestado

6. Ley natural y derecho subjetivo

6.1. Derecho subjetivo en Francisco de Vitoria

6.2. Del derecho natural al derecho de gentes

7. Naturaleza caída, dominio y propiedad en el pensamiento escolástico

8. Dominio y propiedad en Francisco de Vitoria

9. Comentario a la cuestión 66 de la Secunda Secundae, «sobre el hurto y la rapiña»

9.1. Artículo 1: «Si es natural para el hombre la posesión de cosas exteriores»

9.2. Artículo 2: «Si es lícito a alguien poseer una cosa como propia»

9.3. Artículo 3: «Si pertenece a la naturaleza del hurto tomar lo ajeno ocultamente»

9.4. Artículo 4: «Si el hurto y la rapiña son pecados diferentes en especie»

9.5. Artículo 5: «Si el hurto siempre es pecado»

9.6. Artículo 6: «Si el hurto es pecado mortal»

9.7. Artículo 7: «Si es lícito a alguien hurtar por necesidad»

9.8. Artículo 8: «Si la rapiña podría hacerse sin pecado»

9.9. Artículo 9: «Si el hurto es un pecado más grave que la rapiña»

10. Edición bilingüe de los manuscritos:

10.1. Cód. 43/SUV2a/ Biblioteca Universitaria de Salamanca

10.2. OV2A Códice Ottoboniano latino 1015/ Biblioteca Vaticana (Roma)

10.3. MM/ códice 64/ Monasterio de Monserrat (Monserrat)

\section{Bibliografia}

ALONSO GETINO, L. (1933-1935). Relecciones Teológicas. Madrid, España: Asociación Francisco de Vitoria. AQUINO, T. DE (1995). Suma Teológica. Madrid, España: Biblioteca de Autores Cristianos. ARISTÓTELES (2001). Ética a Nicómaco. Madrid, España: Alianza.

BELTRÁN DE HEREDIA, V. (1928). Los Manuscritos del Maestro fray Francisco de Vitoria. Madrid-Valencia, España: Tipografía Moderna.

- (1930). Los manuscritos de los teólogos de la Escuela Salmantina. CienTom, 42, pp. 227-247.

- (1934). Comentarios a la Secunda Secundae. Salamanca, España: Biblioteca de Teólogos Españoles.

- (1939). Francisco de Vitoria. Barcelona, España: Labor.

- (1943). Hacia un inventario analítico de manuscritos teológicos de la Escuela Salmantina, siglos xV-xVII, conservados en España y en el extranjero. Revista Española de Teología, 3, pp. 59-88. 
CARABIAS TORRES, A. M. (2015). La Escuela de Salamanca. Perspectivas de Investigación. The School of Salamanca Working Paper Series, 3, pp. 3-36.

CASTEÑEDA, F. (2010). Francisco de Vitoria. Relección sobre el homicidio y comentario a la cuestión 64 "Sobre el homicidio» de la Suma Teológica II-II, de Tomás de Aquino. Bogotá, Colombia: Universidad de los Andes.

GARCÍA AÑOVEROS, J. (2012). Las ediciones críticas del «Corpus Hispanorum de Pace» del CSIQ. Helmántica, 63, 190, pp. 491-510.

LANGELLA, S. (2011). Teología y ley natural. Estudio sobre las lecciones de Francisco de Vitoria. Madrid, España: Biblioteca de Autores Cristianos.

PEREÑA, L. y PÉREZ PRENDES J. M. (1967). Relectio de Indis o libertad de los Indios. Madrid, España: CSIC/ CHP 5.

PEREÑA, L., MASEDA, F., ABRIL, V. \& BACIERO, Q. (1981). Relectio De iure belli o paz dinámica. Escuela Española de la Paz. Primera generación 1526-1560. Madrid, España: CSIC/ CHP 5.

PIÑEROS, F. (1983). Bibliografía de la Escuela de Salamanca. Pamplona, España: Universidad de Navarra. URDÁNOZ, T. (1960). Obras de Francisco de Vitoria. Relecciones Teológicas. Madrid, España: Biblioteca de Autores Cristianos. 